\title{
Resource utilization and outcomes of intoxicated drivers
}

Robert A Cherry ${ }^{1 *}$, Pamela A Nichols ${ }^{1}$, Theresa M Snavely ${ }^{1}$, Lindsay J Camera² ${ }^{2}$ David T Mauger ${ }^{2}$

\begin{abstract}
Background: The high risk behavior of intoxicated drivers, impaired reaction time, lack of seat belt use, and increased incidence of head injury raises questions of whether pre-hospital use of alcohol leads to a higher injury severity score and worse clinical outcomes. We therefore compared intoxicated and non-intoxicated drivers of motor vehicle crashes with respect to outcome measurements and also describe the resources utilized to achieve those outcomes at our Level 1 trauma center.

Methods: Retrospective descriptive study (Jan 2002-June 2007) of our trauma registry and financial database comparing intoxicated drivers with blood alcohol levels $(B A C)>80 \mathrm{mg} / \mathrm{dl}(\mathrm{ETOH}>80)$ with drivers who had a BAC of $0 \mathrm{mg} / \mathrm{dl}(\mathrm{ETOH}=0)$. Drivers without a BAC drawn or who had levels ranging from $1 \mathrm{mg} / \mathrm{dL}$ to $80 \mathrm{mg} / \mathrm{dL}$ were excluded. Data was collected on demographic information (age, gender, injury severity score or ISS), outcome variables (mortality, complications, ICU and hospital LOS, ventilator days) and resource utilization (ED LOS, insurance, charges, costs, payments). Statistical analysis: $p<0.05$ vs. ETOH $>80$; stratified chi square.

Results: Out of 1732 drivers, the combined study group ( $n=987$ ) of $623 \mathrm{ETOH}=0$ and $364 \mathrm{ETOH}>80$ had a mean age of $38.8 \pm 17.9$, ISS of $18.0 \pm 12.1$, and $69.8 \% \%$ male. There was no difference in ISS ( $p=0.67$ ) or complications $(p=0.38)$. There was a trend towards decreased mortality $(p=0.06)$. The $\mathrm{ETOH}=0$ group had more patients with a prolonged ICU LOS ( $\geq 5$ days), ventilator days ( $\geq 8$ days), and hospital LOS ( $>14$ days) when compared to the ETOH $>80$ group $(p<0.05)$. The ETOH $>80$ group tended to be self pay $(4.9 \%$ vs. $0.7 \%, p<0.5)$ and less likely to generate payment for hospital charges $(p<0.5)$. Hospital charges and costs were higher in the $\mathrm{ETOH}=0$ group $(\mathrm{p}<0.5)$.

Conclusions: The data suggests that intoxicated drivers may have better outcomes and a trend towards reduced mortality. They appeared to be less likely to have prolonged hospital LOS, ICU LOS, and ventilator days. We also observed that intoxicated drivers were more likely to be self-pay, less likely to have charges $>\$ 50 \mathrm{~K}$, and less likely to pay $\geq 90 \%$ of the charges. Further research using multivariable analysis is needed to determine if these apparent outcomes differences are driven by acute intoxication, and the tendency for endotracheal intubation and ICU admission, rather than injury severity.
\end{abstract}

\section{Background}

The prevalence of motor vehicle crashes remains a major public health concern and is a leading cause of morbidity and mortality in the United States. There were 38,588 fatalities and 1,746,000 injuries associated with motor vehicle crashes that were reported by police in 2006 [1]. Many of these motor vehicle crashes are alcohol-related and accounted for 13,470 fatalities [2]. The National

\footnotetext{
* Correspondence: rcherry@psu.edu

'Penn State Milton S. Hershey Medical Center, Department of Surgery, Shock Trauma Center, Hershey, Pennsylvania 17033, USA

Full list of author information is available at the end of the article
}

Highway Traffic Safety Administration (NHTSA) has reported that $20 \%$ of fatal crashes in 2005 involved drunk drivers (BAC of $\geq 80 \mathrm{mg} / \mathrm{dL}$ ) [3]. According to NHTSA, alcohol-related crashes account for $\$ 51.1$ billion or $22 \%$ of the economic costs. Approximately $75 \%$ of these costs involve crashes in which a driver or non-occupant had a BAC of at least $100 \mathrm{mg} / \mathrm{dl}$. The high percentage of intoxicated drivers is therefore a major driver of the socioeconomic costs involving motor vehicle crashes.

Moreover, there have been a number of studies that have found an association between drinking and driving and an increased risk for motor vehicle injury [4-6]. The 
Insurance Institute for Highway Safety reported that a $\mathrm{BAC}$ as low as $20 \mathrm{mg} / \mathrm{dL}$ increases the likelihood of a crash while operating a motor vehicle [5]. The probability of a crash increases significantly at $50 \mathrm{mg} / \mathrm{dL}$ and rises rapidly at levels greater than $100 \mathrm{mg} / \mathrm{dL}$. Even among people who are 55 years and older, drinking history (12 or more drinks prior to death) is associated with fatality from a motor vehicle crash [7].

The incidence of alcohol-impaired driving, and the risk of injury from alcohol-related motor vehicle crashes, is also strongly associated with binge drinking $[8,9]$. Other predictors for a recurrent motor vehicle crash included age $<32$ years old, male sex, nighttime crash, and a BAC > $50 \mathrm{mg} / \mathrm{dl}$ [6]. Drivers who tested positive for blood alcohol are noncompliant with seat belt use [10-13] and are more likely to have suffered a head injury $[11,13,14]$.

The high risk behavior of intoxicated drivers, impaired reaction time, lack of seat belt use, and increased incidence of head injury all raises the question of whether pre-hospital use of alcohol leads to a higher injury severity score, and therefore worse clinical outcomes. We therefore compared intoxicated and non-intoxicated drivers of motor vehicle crashes with respect to outcome measurements and also describe the resources utilized to achieve those outcomes at our Level 1 trauma center.

\section{Methods}

This was a retrospective descriptive study conducted at our Level 1 trauma center from January 2002 through June 2007. Adult trauma patients who were 18 years of age and older, operated a motor vehicle, and involved in a crash were identified using our trauma registry (Collector, Digital Innovation, Forest Hill, Maryland). Drivers were identified and differentiated from passengers based on EMS and/or emergency department records. Patients with documented blood alcohol tests were identified by accessing the electronic medical record (Cerner Corporation, Kansas City, Missouri). There is mandatory reporting of all suspected or confirmed cases of alcohol intoxication to the Pennsylvania Department of Transportation. At our institution, blood alcohol concentrations are obtained on suspected cases of alcohol intoxication if the laboratory finding might affect therapeutic decision-making or result in a referral to a drug and alcohol counselor.

We compared intoxicated drivers with alcohol concentrations $>80 \mathrm{mg} / \mathrm{dl}(\mathrm{ETOH}>80)$ with drivers who had alcohol concentrations of $0 \mathrm{mg} / \mathrm{dl}(\mathrm{ETOH}=0)$. Drivers without an ETOH level drawn or who had results ranging from $1 \mathrm{mg} / \mathrm{dl}$ to $80 \mathrm{mg} / \mathrm{dl}$ were excluded from the study. The legal limit for blood alcohol concentrations in Pennsylvania is $80 \mathrm{mg} / \mathrm{dL}$. Patients without a blood alcohol concentration drawn and inter-facility transfers were excluded.

Data was collected on outcome measurements such as mortality, complications, ICU and hospital length of stay or LOS, and ventilator days. Resource utilization was assessed by obtaining variables on time spent in the emergency department (ED), insurance type, inpatient charges, medical costs, and payments made (PMT). Information on discharge destination from the ED and the hospital was also collected. The Cochran-MantelHaenszel Chi-square test, stratified by age, gender, and injury severity score (ISS), was used to compare the $\mathrm{ETOH}>80$ group against the $\mathrm{ETOH}=0$ group with respect to these outcomes.

All analyses were carried out using SAS Version 9 (SAS Institute Inc., Cary, NC). All p values $<0.05$ were used to denote significant differences between groups. The Cochran-Mantel-Haenszel Chi-square test, stratified by age, gender, and injury severity score (ISS), was used to compare the ETOH $>80$ group against the ETOH = 0 group with respect to these outcomes and odds ratios were calculated for binary outcomes. Both stratified and unstratified odds ratios with $95 \%$ confidence intervals were constructed. The study protocol was approved by the Institutional Review Board at the Penn State Milton S. Hershey Medical Center.

\section{Results}

There were 1,732 adult drivers of motor vehicles identified during the study period who were evaluated at our trauma center. The combined study group consisted of 623 patients with a $\mathrm{BAC}$ of $0 \mathrm{mg} / \mathrm{dL}(\mathrm{ETOH}=0$ group) and 364 patients with a $\mathrm{BAC}>80 \mathrm{mg} / \mathrm{dL}(\mathrm{ETOH}>80$ group). Collectively, these patients had a mean age of $38.8 \pm 17.9$ years, an ISS of $18.0 \pm 12.1$, and were $69.8 \%$ male. There was no significant difference is ISS among the two groups $(\mathrm{p}<0.66)$, but the $\mathrm{ETOH}>80$ group was younger and had more men $(\mathrm{p}<0.05)$.

The ETOH $=0$ group tended to have a prolonged ED LOS ( $\geq 220$ minutes, see Table 1 ). The ETOH $>80$ group was more likely to be admitted to the ICU $(41.3 \%$ vs. $33.7 \%, \mathrm{p}<0.05)$ and intermediate care unit $(18.2 \%$ vs. $16.1 \%, \mathrm{p}<0.05)$ from the ED, and the $\mathrm{ETOH}=0$ group was more likely to go to the operating room (Table 2). Despite fewer admissions to the ICU, the ETOH $=0$ group had significantly prolonged ICU LOS ( $\geq 5$ days), ventilator days ( $\geq 8$ days), and hospital LOS ( $>14$ days) when compared to the ETOH $>80$ group $(\mathrm{p}<0.05)$ (Table 2). There was no significant difference in the number of deaths for the ETOH $>80$ group, but there was a trend towards decreased mortality $(\mathrm{p}=0.06)$.

The ETOH > 80 group tended to be self pay (4.9\% vs. $0.7 \%, \mathrm{p}<0.5)$ and less likely to generate payment for hospital charges $(\mathrm{p}<0.5)$ (Table 3$)$. Of note, hospital 
Table 1 Outcome measurements of intoxicated vs. nonintoxicated drivers

\begin{tabular}{ccccc}
\hline & $\begin{array}{c}\mathrm{ED} \geq \mathbf{2 2 0} \\
\text { min. }\end{array}$ & $\begin{array}{c}\text { ICU LOS } \\
\mathbf{2} \text { 5days }\end{array}$ & $\begin{array}{c}\text { Vent days } \\
\mathbf{2}\end{array}$ & $\begin{array}{c}\text { Hospital LOS } \\
>\mathbf{1 4} \text { days }\end{array}$ \\
\hline $\mathrm{ETOH}=0$ & $25.6 \%^{*}, 159 /$ & $19.0 \%^{*}, 118 /$ & $10.5 \%^{*}, 65 /$ & $17.8 \%^{*}, 111 /$ \\
$\mathrm{ETOH}>$ & $22.5 \%, 78 / 346$ & $13.5 \%, 49 / 364$ & $5.0 \%, 18 / 361$ & $11.5 \%, 42 / 364$
\end{tabular}
80

* $-\mathrm{p}<0.05$ vs. ETOH $>80$

ED - emergency department

ICU - intensive care unit

Vent - ventilator

LOS-length of stay

$\mathrm{ETOH}=0$-blood alcohol level of $0 \mathrm{mg} / \mathrm{dL}$

$\mathrm{ETOH}>80$

blood alcohol level $>80 \mathrm{mg} / \mathrm{dL}$

charges and medical costs were significantly higher in the $\mathrm{ETOH}=0$ group $(\mathrm{p}<0.5)$. The $\mathrm{ETOH}>80$ group was significantly more likely to go home $(76.5 \%$ vs. $65.6 \%)$ and less likely to be discharged to a skilled nursing facility (1.5\% vs. $5.2 \%)$ and a rehabilitation center (15.7\% vs. $25.6 \%)$ (Table 4$)$.

\section{Discussion}

Our study describes several unexpected observations among intoxicated drivers with respect to resource utilization and outcome measurements. These results underscore several areas requiring further discussion, including the burden that alcohol misuse and abuse may place on hospital resource utilization, and whether alcohol intoxication is a potential predictor of clinical outcome. In addition, the financial implications that intoxicated drivers have on our Level 1 trauma center becomes more of an issue after reviewing the data in this study.

Although our study did not specifically control for ISS, the data did not show differences in ISS between BAC > 80 and $\mathrm{BAC}=0$ groups. The groups were also comparable in that there were no differences in complications or mortality. Nevertheless, there have been conflicting reports in the literature with regards to injury severity for occupants involved in alcohol-related motors vehicles crashes. Ward and his colleagues, for instance,
Table 3 Financial variables of intoxicated vs. nonintoxicated drivers

\begin{tabular}{lcccc}
\hline Self-pay & $\begin{array}{c}\text { Charges }> \\
\mathbf{5 0 K}\end{array}$ & $\begin{array}{c}\text { Costs }> \\
\mathbf{5 2 5 K}\end{array}$ & $\begin{array}{c}\text { PMT/Charge }> \\
\mathbf{0 . 9}\end{array}$ \\
$\mathrm{ETOH}=$ & $0.7 \%^{*}, 3 /$ & $29.6 \% \%^{*}, 123 /$ & $25.5 \%, 106 /$ & $28.4 \%^{*}, 118 / 416$ \\
0 & 416 & 416 & 416 & \\
\hline $\mathrm{ETOH}>$ & $4.9 \%, 10 /$ & $20.8 \%, 42 / 202$ & $18.1 \%, 38 /$ & $16.3 \%, 33 / 202$ \\
80 & 203 & & 202 & \\
\hline
\end{tabular}

* $-\mathrm{p}<0.05$ vs. ETOH $>80$

PMT - payment

$\mathrm{ETOH}=0$ - blood alcohol level of $0 \mathrm{mg} / \mathrm{dL}$

$\mathrm{ETOH}>80$

blood alcohol level $>80 \mathrm{mg} / \mathrm{dL}$

studied 1,198 trauma patients with evidence of alcohol use and found no difference in the severity of injury compared with those with a negative blood alcohol concentrations [15]. In another study, Smink and associates also found no difference in the mean ISS scores between drivers who test negative for alcohol, and those who do not [16]. On the other hand, Brown et. al. found that ISS was significantly higher for occupants of vehicles that were operated by intoxicated drivers with a blood alcohol concentrations of $100 \mathrm{mg} / \mathrm{dl}$ [17]. Honkanen and his associates also found a positive correlation between injury severity and intoxication among injured occupants of motor vehicles [18].

The issue that emerges is whether there are outcome differences between intoxicated drivers regardless of ISS. Shih and his colleagues investigated 923 injured drivers involved in motor vehicle and motorcycle crashes in which 421 of them had BAC $\geq 50$ [19]. Drivers with a $\mathrm{BAC} \geq 50$ had a significantly higher ISS. However, after a logistic regression analysis, a BAC $\geq 50$ was not associated with severe injury as defined by an ISS $\geq 9$ or mortality. However, alcohol intoxication $(B A C \geq 50)$ was a predictor of morbidity. In the Washington State study by Mueller, BAC > $50 \mathrm{mg} / \mathrm{dL}$ was also found to be a predictor for morbidity [20]. Age greater than 54 years old and BAC > $50 \mathrm{mg} / \mathrm{dL}$ did not predict mortality.

Perhaps one of the more provocative studies was performed by Koval and associates. They performed a recent retrospective study of 67,021 patients in which

Table 2 Post emergency department destination

\begin{tabular}{lccccc}
\hline & ICU & Medical/Surgical Unit & Intermediate Care Unit & OR & Morgue \\
\hline $\mathrm{ETOH}=0$ & $33.7 \%, \mathrm{n}=209$ & $28.0 \%, \mathrm{n}=174$ & $16.1 \%, \mathrm{n}=100$ & $22.1 \%, \mathrm{n}=137$ & $0.2 \%, \mathrm{n}=1$ \\
$\mathrm{ETOH}>80$ & $41.3 \%, \mathrm{n}=150$ & $21.8 \%, \mathrm{n}=79$ & $18.2 \%, \mathrm{n}=66$ & $17.9 \%, \mathrm{n}=65$ & $0.8 \%, \mathrm{n}=3$ \\
Total & 359 & 253 & 166 & 202 & 4 \\
\hline
\end{tabular}

ICU - intensive care unit

OR - operating room

LOS- length of stay

$\mathrm{ETOH}=0$ - blood alcohol level of $0 \mathrm{mg} / \mathrm{dL}$

$\mathrm{ETOH}>80$

blood alcohol level $>80 \mathrm{mg} / \mathrm{dL}$ 
Table 4 Most common discharge destinations

\begin{tabular}{lcccccc}
\hline & Home & Rehabilitation Center & Skilled Nursing Facility & Legal Authority & $\begin{array}{c}\text { AMA } \\
\text { Hospital Transfer }\end{array}$ & $\begin{array}{c}\text { Psychiatric } \\
\text { Facility }\end{array}$ \\
\hline $\mathrm{ETOH}=0$ & $65.6 \%, \mathrm{n}=395$ & $25.6 \%, \mathrm{n}=154$ & $5.2 \%, \mathrm{n}=31$ & $0.2 \%, \mathrm{n}=1$ & $0.5 \%, \mathrm{n}=3$ & $1.0 \%, \mathrm{n}=6$ \\
$\mathrm{ETOH}>80$ & $76.5 \%, \mathrm{n}=264$ & $15.7 \%, \mathrm{n}=54$ & $1.5 \%, \mathrm{n}=5$ & $2.0 \%, \mathrm{n}=7$ & $1.5 \%, \mathrm{n}=5$ & $0.3 \%, \mathrm{n}=1$ \\
Total & 659 & 208 & 36 & 8 & $7.5 \%, \mathrm{n}=5$ \\
\hline
\end{tabular}

AMA - against medical advice

$\mathrm{ETOH}=0$ - blood alcohol level of $0 \mathrm{mg} / \mathrm{dL}$

$\mathrm{ETOH}>80$

blood alcohol level $>80 \mathrm{mg} / \mathrm{dL}$

38.3\% were drivers involved in a MVC [11]. The strongest predictor for mortality was ISS. Other risk factors cited were male sex and age. Most notably, "protective factors" included the presence of alcohol and the use of safety devices. Other published reports also suggest that alcohol intoxication may be protective. For example, outcomes after MVC and isolated severe traumatic brain injury are significantly different depending upon the level of ETOH and not simply upon the presence or absence of serum ETOH [21,22].

One should not necessarily infer from the data described in our study, however, that intoxication is protective for drivers involved in a motor vehicle crash. This study is limited in that logistic regression analysis was not performed. The culling of additional numerical or categorical predictor variables to determine the probability of occurrence is certainly desirable. We believe that a multivariable analysis to determine outcomes differences would best be performed as a prospective study because of the limitations inherent in a retrospective, registry-based investigation. The prospective collection of variables such as vehicle speed and type, daytime vs. nighttime vehicle operation, seat belt use, airbag deployment, driver experience, and distractions (ex. number of occupants) would also be valuable. For these reasons, we have categorized this report as a retrospective descriptive study. Apparent associations due to differences between groups must therefore be interpreted with caution.

Responsibility demands a critical analysis of these observations looking for alterative explanations that require further investigation. For instance, there were an increased number of admissions to the ICU and IMC among the ETOH $>80$ group despite no differences in ISS. This contradiction could be explained if a disproportionate number of intoxicated patients required endotracheal tube intubation and a short ICU length of stay due to binge drinking. Patient care would therefore be driven by medical necessity and not by injury severity. The shorter ICU and hospital LOS found in the ETOH $>80$ is consistent with this line of reasoning. This argument would suggest that some ICU and IMC admissions among patients with an ETOH $>80$ may have been unnecessary. On the other hand, one would have expected to find differences in injury severity score among the two groups if this were the case. Of note, patients with an ETOH $=0$ were more likely to go to the operating room and may have been more seriously injured than what was captured by ISS. This might explain why the ETOH $>80$ group had fewer admissions to the ICU, and a reduced incidence of prolonged mechanical ventilation.

In our data, ED LOS was also notably shorter in the ETOH $>80$ group. The overall relationship between LOS and alcohol intoxication is conflicting in the literature. Brotman and associates found that hospital length of stay among trauma patients who tested positive for alcohol was similar to those who tested negative [23]. In contrast, Mueller found that drivers under the influence of alcohol had longer hospital stays after adjusting for age, gender, and injury severity [20].

We also observed that the ETOH $>80$ group was more likely to be self-pay, more likely to be admitted to the ICU and IMC, and less likely to pay for the charges associated with their medical care. Our trauma center is therefore less likely to recover operating costs when caring for intoxicated drivers. If these findings are consistent at other institutions, then the additional services rendered may represent another financial burden for trauma centers, especially those that are already financially troubled. Of note, Mueller and colleagues has already shown that hospital charges in Washington State were greater for drinking drivers when compared to nondrinking divers [20].

There are several other limitations of this study. It is a retrospective study and contains some missing data elements that are inherent to trauma registries in general. There is also a possibility that patients with a BAC $=0$ are less likely to be admitted to the hospital than intoxicated patients. This would result in a relatively greater resource utilization among patients with an ETOH $>80$. In addition, patients who are discharged directly from the ED are not considered state qualifiers for inclusion in the registry. This may have an impact on ISS between groups, especially if $\mathrm{BAC}=0$ patients are disproportionately discharged. In addition, because only those 
patients that were considered registry qualifiers in our state were included, we do not know the actual number of patients that may have been treated and released, with or without a trauma evaluation. Therefore, we do not know the actual ICU days per patient for either group. Furthermore, alcohol screening was not mandatory for all drivers and might introduce a selection bias into the methodology. Finally, since this is a single center study, our findings may not necessarily reflect those seen at other trauma centers.

Further research is needed in this area. For instance, population-based studies that investigate the outcomes of all drivers in a region, including those dead at the scene, would be valuable. Investigations may also be conducted that carefully control for injury mechanism, such as speed, impact location, type of vehicle, and seat belt/air bag protection. Future studies should also consider selecting a primary outcome parameter of interest. For example, propensity scores could be developed and used to predict the need for intubation and prolonged mechanical ventilation based on age, gender, ISS, abbreviated injury score for the head, Glasgow Coma Score, admission blood pressure and arterial $\mathrm{pO}_{2}$ (or oxygen saturation), and the presence or absence of rib fractures. Intoxicated patients could then be case-matched with unintoxicated patients having similar propensity scores and assessed for the incidence of intubation and prolonged mechanical ventilation. The sample size needed for such a study is probably too small to be conducted at any single institution.

\section{Conclusions}

Drivers of motor vehicle crashes with a BAC $>80 \mathrm{mg} /$ $\mathrm{dL}$ who presented at our trauma center were observed to have better outcomes compared with drivers having no evidence of alcohol use. Intoxicated drivers were described as having lower ICU admission rates, hospital and ICU length of stay, ventilator days, and inpatient charges. Those drivers were also more likely to be discharge to home. The appearance of improved outcomes in intoxicated drivers may have been driven by acute intoxication, and the tendency for endotracheal intubation and ICU admission, rather than injury severity. Further studies are needed to corroborate these observations at other trauma centers.

\section{Additional note}

The authors are pleased that an invited editorial by Dr. Uli Schmucker [24] has been included with this article. Many of his remarks have been considered during the course of our clinical investigation. We agree with his assessment that further research is needed to determine if acute alcohol intoxication has an impact on trauma outcomes.

\section{Acknowledgements}

Poster presentation at Sixty-Seventh Annual Meeting of the American Association for the Surgery of Trauma in Maui, Hawaii, September 24-27, 2008.

\section{Author details}

${ }^{1}$ Penn State Milton S. Hershey Medical Center, Department of Surgery, Shock Trauma Center, Hershey, Pennsylvania 17033, USA. ${ }^{2}$ Penn State Milton S. Hershey Medical Center, Public Health Sciences, Hershey, Pennsylvania 17033, USA.

\section{Authors' information}

RAC: Study concept and design. RAC, PAN, TMS: Acquisition of data. RAC, DTM, LJC: Analysis and interpretation of data. RAC: Drafting of the manuscript. RAC, PAN, TMS, LJC: Critical revision of the manuscript for important intellectual content. DTM, LJC: Statistical analysis. RAC, PAN, TMS: Administrative, technical, and material support. All authors have read and approved the final manuscript.

\section{Competing interests}

The authors declare that they have no competing interests.

Received: 28 December 2009 Accepted: 5 August 2010

Published: 5 August 2010

\section{References}

1. U. S. Department of Transportation: Traffic safety facts 2006 . A compilation of motor vehicle crash data from the fatality analysis reporting system and the general estimate system. 2006 [http://wwwnrd.nhtsa.dot.gov/Pubs/TSF2006FE.PDF], Accessed August 22, 2009.

2. U.S. Department of Transportation: Traffic safety facts 2008 data. 2008 [http://www-nrd.nhtsa.dot.gov/Pubs/811162.PDF], Accessed August 10, 2009.

3. U.S. Department of Transportation: Traffic safety facts 2005. A compilation of motor vehicle crash data from the fatality analysis reporting system and the general estimate system. 2005 [http://www-nrd.nhtsa.dot.gov/ Pubs/TSF2005.PDF], Accessed August 2, 2009.

4. Bell NS, Amoroso PJ, Yore MM, Smith GS, Jones BH: Self-reported risktaking behaviors and hospitalization for motor vehicle injury among active duty army personnel. Am J Prev Med 2000, 18:85-95.

5. Insurance Institute for Highway Safety (IIHS): Fatality facts. Arlington, VA 1993.

6. Fabbri A, Marchesini $G$, Dente $M$, lervese $T$, Spada M, Vandelli A: A positive blood alcohol concentration is the main predictor of recurrent motor vehicle crash. Ann Emerg Med 2005, 46:161-167.

7. Sorock GS, Chen LH, Gonzalgo SR, Baker SP: Alcohol-drinking history and fatal injury in older adults. Alcohol 2006, 40:193-199.

8. Quinlan KP, Brewer RD, Siegel P, et al: Alcohol-impaired driving among U. S. adults, 1993-2002. Am J Prev Med 2005, 28:346-350.

9. Vingilis $E$, Wilk P: Predictors of motor vehicle collision injuries among a nationally representative sample of Canadians. Traffic Inj Prev 2007, 8:411-418.

10. Allen S, Zhu S, Sauter C, Layde P, Hargarten S: A comprehensive statewide analysis of seatbelt non-use with injury and hospital admissions: new data, old problem. Acad Emerg Med 2006, 13:427-434.

11. Koval KJ, Cooley M, Cantu RV, Spratt KF: The effects of alcohol on inhospital mortality in drivers admitted after motor vehicle accidents. Bull NYU Hosp Jt Dis 2008, 66:27-34.

12. Ball CG, Kirkpatrick AW, Brenneman FD: Noncompliance with seat-belt use in patients involved in motor vehicle collisions. Can J Surg 2005, 48:367-372.

13. Honkanen R, Smith G: Impact of acute alcohol intoxication on patterns of non-fatal trauma: cause-specific analysis of head injury effect. Injury 1991, 22:225-229.

14. Chen SC, Lin FY, Chang KJ: Body region prevalence of injury in alcoholand non-alcohol-related traffic injuries. J Trauma 1999, 47:881-884.

15. Ward RE, Flynn TC, Miller PW, Blaisdell WF: Effects of ethanol ingestion on the severity and outcome of trauma. Am J Surg 1982, 144:153-157.

16. Smink BE, Movig KL, Lusthof KJ, De Gier JJ, Uges DR, Egberts AC: The relation between the use of psychoactive substances and the severity of 
the injury in a group of crash-involved drivers admitted to a regional trauma center. Traffic Inj Prev 2008, 9:105-108.

17. Brown CK, Cline DM: Factors affecting injury severity to rear-seated occupants in rural motor vehicle crashes. Am J Emerg Med 2001, 19:93-98.

18. Honkanen R, Smith GS: Impact of acute alcohol intoxication on the severity of injury: a cause-specific analysis of non-fatal trauma. Injury 1990, 21:353-357.

19. Shih HC, Hu SC, Yang CC, Ko TJ, Wu JK, Lee CH: Alcohol intoxication increases morbidity in drivers involved in motor vehicle accidents. Am J Emerg Med 2003, 21:91-94.

20. Mueller BA, Kenaston T, Grossman D, Salzberg P: Hospital charges to injured drinking drivers in Washington State: 1989-1993. Accid Anal Prev 1998, 30:597-605.

21. Plurad D, Demetriades D, Cruzinski G, et al: Motor vehicle crashes: the association of alcohol consumption with the type and severity of injuries and outcomes. J Emer Med 2010, 38:12-17.

22. Talving $P$, Plurad $D$, Barmparas $G$, et al: Isolated severe traumatic brain injuries: Association of blood alcohol levels with the severity of injuries and outcomes. J Trauma 2010, 68:357-362.

23. Brotman S, Indeck MC, Leonard D, Huber J: The study of the relationship between lifestyle characteristic self-reported drinking patterns and trauma. Am Surg 1995, 61:975-979.

24. Schmucker U: Editorial Commentary: Resource utilization and outcomes of intoxicated drivers: does evidence of alcohol-impaired driving affect road traffic crash injury outcomes? Journal of Trauma Management and Outcomes 2010.

doi:10.1186/1752-2897-4-9

Cite this article as: Cherry et al.: Resource utilization and outcomes of intoxicated drivers. Journal of Trauma Management \& Outcomes 2010 4:9.

\section{Submit your next manuscript to BioMed Central and take full advantage of:}

- Convenient online submission

- Thorough peer review

- No space constraints or color figure charges

- Immediate publication on acceptance

- Inclusion in PubMed, CAS, Scopus and Google Scholar

- Research which is freely available for redistribution

Submit your manuscript at www.biomedcentral.com/submit 